\title{
Evolución clínica de trabajadores con fractura de radio distal Fernández III. Tratamiento no complicado quirúrgico y no quirúrgico
}

\author{
Clinical evolution of workers with Fernández III distal radius fracture. Surgical and \\ non-surgical non-complicated treatment
}

\author{
Arturo García-Galicia', Juan S. Rueda-Mojica², Miguel A. Sánchez-Durán², \\ Rodolfo G. Barragán-Hervella², Álvaro J. Montiel-Jarquín ${ }^{1 *}$ y Suemmy Gaytán-Fernández² \\ ¿Unidad Médica de Alta Especialidad Hospital de Especialidades de Puebla, Centro Médico Nacional General de División Manuel Ávila Camacho, \\ Instituto Mexicano del Seguro Social (IMSS); ²Unidad Médica de Alta Especialidad Hospital de Traumatología y Ortopedia de Puebla, IMSS. Puebla, \\ México
}

\section{Resumen}

Antecedentes: Las fracturas de radio distal Fernández III (FRDF III) son una urgencia muy frecuente. Las indicaciones quirúrgicas y no quirúrgicas persisten controversiales, así como el impacto funcional. Objetivo: Comparar la evolución clínica de Ios trabajadores con FRDF III, con manejo quirúrgico frente a no quirúrgico, no complicados, en un hospital especializado. Método: Estudio comparativo, observacional y longitudinal en trabajadores con FRDF III unilateral cerrada, de ambos sexos, con evolución menor de 5 días. Se excluyeron pacientes con comorbilidad afectante de consolidación ósea y polifracturados. Se eliminaron aquellos con información incompleta, con complicaciones, con aparato de yeso modificado 5 días después de su colocación, con mala reducción-estabilización acorde a la AO. Se aplicó la Escala Funcional de Mayo para Muñeca (EFMM) a los 3 y 6 meses del tratamiento. Se utilizó la prueba t de Student. Resultados: 159 pacientes, 34\% mujeres, 66\% hombres. Edad promedio 40.18 años. Manejo quirúrgico 42,5\% (placa 8.7\%, fijador 29\%, fijador + clavillo 8.7\%); no quirúrgico $33 \%$. Diferencias significativas ( $p \leq 0.05$ ): EFMM 3 y 6 meses; a 3 meses: dolor, prensión; a 6 meses: movilidad; y días totales de incapacidad. Conclusiones: El tratamiento quirúrgico muestra mejores resultados funcionales, destacando el dolor y la prensión a 3 meses, y la movilidad a 6 meses, con reincorporación laboral en menos tiempo.

Palabras clave: Fractura. Radio distal. Fernández III. Tratamiento. Incapacidad laboral.

\begin{abstract}
Background: This fracture is a very frequent emergency. Surgical and non-surgical management are still controversial, such as functional impact. Objective: To compare clinical evolution of Fernandez III distal radius fractures, managed with surgically vs no surgical treatment, in a specialized hospital. Method: Comparative, observational, longitudinal study, released on workers with unilateral Fernandez III closed distal radius fractures, both sex, with evolution less than 5 days. Those patients with pathologies getting difficult in bone consolidation, and with several fractures were excluded. Those with incomplete information, complicated, and whom paste were modified after 5 days, and with bad reduction or stabilization fracture according AO were eliminated. Mayo Functional Wrist Scale (EFMM) was applied at 3 and 6 months. Student-t test was applied.

\section{Correspondencia:}

*Álvaro J. Montiel-Jarquín

Calle 2 norte 2004

Col. Centro

Fecha de recepción: 07-05-2020

C.P. 72000, Puebla, Pue., México

E-mail: dralmoja@ hotmail.com

0009-7411/@ 2020 Academia Mexicana de Cirugía. Publicado por Permanyer. Este es un artículo open access bajo la licencia CC BY-NC-ND (http://creativecommons.org/licenses/by-nc-nd/4.0/).

Cir Cir. 2021;89(3):377-383

Contents available at PubMed www.cirugiaycirujanos.com
\end{abstract}


Results: 159 patients recruited, 34\% females, 66\% males. Medium age 40 years old. Surgical treatment $42.5 \%$ (plaque $8.7 \%$, external fixation $29 \%$, external fixation and nails $8.7 \%$ ). Non-surgical treatment $33 \%$. Significative differences ( $p \leq 0.05)$ : EFMM at 3 and 6 months; pain, grasp at 3 months; mobility at 6 months; total laboral inhability days. Conclusions: Surgical treatment shows better functional results, specially pain and grasp at 3 months, mobility at 6 months, and faster returning to work.

Key words: Fracture. Distal radius. Fernandez III. Treatment. Laboral inhability

\section{Introducción}

La fractura de radio distal es una de las fracturas más frecuentes reportadas en la literatura internacional ${ }^{1-4}, y$ especialmente entre la población trabajadora ${ }^{2}$. Se reportan hasta 640,000 casos anuales en los Estados Unidos Unidos de Norteamérica, siendo la sexta más frecuente. Predomina en edades de 40 a 60 años; en la población pediátrica y juvenil prevalece el sexo masculino, y en población adulta el femenino, y hacia los 50 años de edad la relación mujer/hombre se iguala ${ }^{4-6}$.

Existen múltiples clasificaciones, por lo que se pueden encontrar dos o más procedimientos para el tratamiento $0^{1,2,4}$.

Respecto a la producción de la fractura, se consideran las siguientes formas biomecánicas ${ }^{3,7-9}$ :

- Mecanismo de carga axial, sobre la cara articular distal del radio, carga de características indirectas.

- Asociado a mano en hiperextensión, entre 40 y 90 grados.

Esto se traduce en fuerzas de tensión en la cara volar y de compresión en la cara dorsal.

Las proyecciones radiológicas útiles para el diagnóstico son la anteroposterior, la lateral y las oblicuas. Estudios adicionales, como la tomografía computarizada y la resonancia nuclear, no muestran diferencias para diagnosticar estas fracturas 1,10-12. $^{12}$.

La clasificación de Fernández se basa en el mecanismo de la lesión más que solo en datos radiográficos, y evalúa la afectación ósea y de partes blandas (ligamentos), lo que modifica el pronóstico ${ }^{3}$. Comprende cinco grupos (Fig. 1).

Los objetivos generales del tratamiento se centran en restablecer la congruencia articular, así como los ejes metáfisis-diáfisis del radio distal. Por lo tanto, se deben conocer las características del trazo de fractura, la inestabilidad del trazo, las lesiones asociadas y la funcionalidad del paciente $e^{4,7,11,13-15}$.

En la ligamentotaxis, la tracción y la contracción se transmiten a los ligamentos extrínsecos de la articulación radio-carpal y radio-ulnar, y permite movilizar los fragmentos para que después se fijen. La técnica de reducción cerrada está ampliamente descrita en diversos reportes; incluye el manejo del dolor, maniobras de reducción, inmovilización con escayola y control radiográfico $8,16,17$.

El objetivo de nuestro estudio fue analizar la evolución clínica y la incapacidad laboral de pacientes trabajadores con fractura de radio distal Fernández III, manejados sin complicaciones en el Hospital de Traumatología y Ortopedia de Puebla (HTOP), del Instituto Mexicano del Seguro Social (IMSS), durante 6 meses, mediante la Escala Funcional de Mayo para Muñeca (EFMM).

\section{Método}

El presente trabajo fue aprobado por el Comité Local de Investigación y Ética en Investigación en Salud No. 2105 del IMSS con el registro R-20172105-29. Se realizó un estudio comparativo, observacional y longitudinal en el HTOP del IMSS. Se incluyeron los pacientes con fractura de Fernández III que firmaron el consentimiento informado, de ambos sexos, trabajadores, con una evolución máxima de 5 días de la fractura a la fecha de atención, con fractura unilateral, cerrada y manejo sin complicaciones. Se excluyeron los pacientes con enfermedades crónicas degenerativas que afectan la consolidación ósea (diabetes descompensada, enfermedad renal crónica, hipotiroidismo, osteoporosis, osteopenia, artritis reumatoide) y politraumatizados. Se eliminaron los pacientes que solicitaron salir del estudio, los que no completaron la información requerida y aquellos con presencia de lesión vascular, lesión de nervio periférico, infección, reintervención, etc.; también los pacientes que recibieron modificación del aparato de yeso después de 5 días de su colocación y aquellos en que la reducción y estabilización hubiese sido mala según los criterios establecidos en la AO.

Todos los pacientes fueron evaluados al mes del tratamiento, cuando fue posible referir al protocolo de rehabilitación para iniciar la movilización a aquellos que presentaron adecuada consolidación (el 


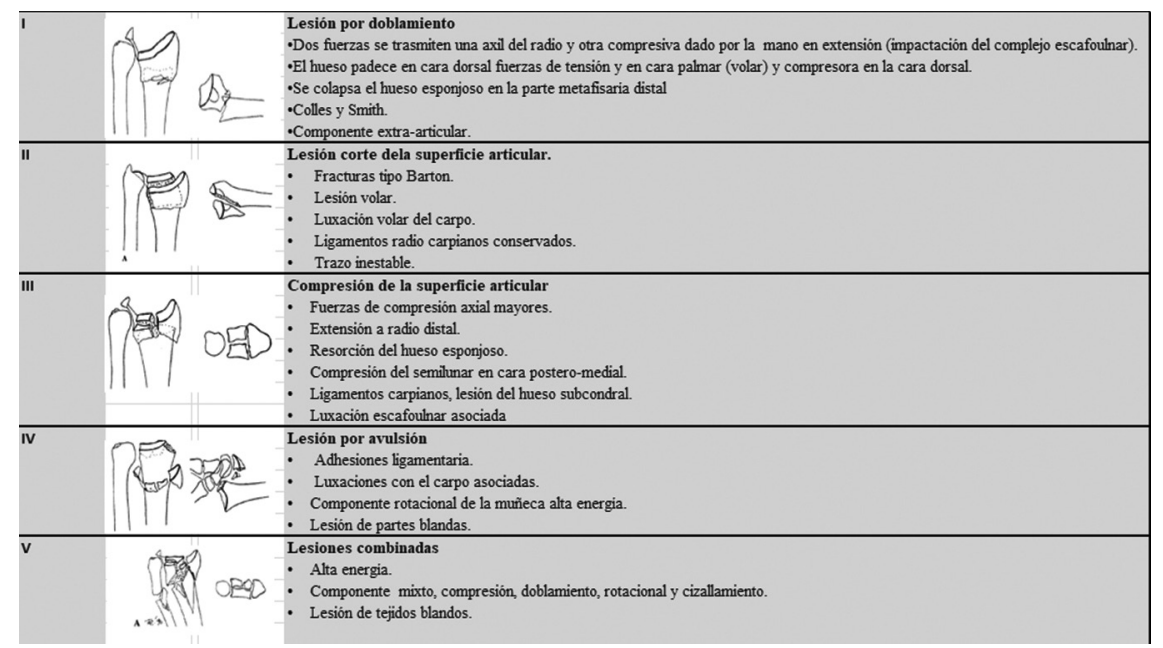

Figura 1. Clasificación de Fernández. (Elaborada con información e imágenes de Fernandez D, Jupiter J. Fractures of the distal radius. New York: Springer; 1996).

promedio de consolidación de cualquier fractura es de 30 días), con el propósito de rescatar rangos de movilidad, desinflamación y posteriormente recuperación de la fuerza. Recibieron una consulta de seguimiento a los 2 meses para verificar y reforzar el cumplimiento del programa de rehabilitación.

Se registró la información del expediente y directamente de la exploración de los pacientes y las radiografías. Los pacientes se evaluaron con la EFMM (que evalúa dolor, estado laboral, rango de movilidad, fuerza de agarre o prensión) al tercer y sexto meses posteriores al tratamiento ${ }^{18,19}$.

Se utilizó la prueba t de Student para comparar la funcionalidad y se consideró significativo un valor de $p \leq 0.05$.

\section{Resultados}

Ingresaron 115 pacientes con manejo no quirúrgico, de los cuales se eliminaron 39 (34\%) de acuerdo con los criterios de selección: uno por presentación de síndrome compartimental y 38 por edema que se resolvieron con remanipulación del yeso inicial, y registrándose hasta 25 pacientes con colapso de la fractura, que requirieron manejo quirúrgico con fijadores. Del manejo quirúrgico se reclutaron 86 pacientes y se eliminaron $3(3.5 \%)$ : uno por lesión de la arteria radial durante la aplicación de la placa, quien requirió reparación por cirugía reconstructiva, y dos pacientes más que presentaron síndrome doloroso regional, por lo que se modificó la posición de la muñeca en la inmovilización.
Finalmente fueron estudiados 159 pacientes; de ellos, 83 (52\%) con manejo quirúrgico. La mayoría $(66 \%)$ eran hombres. La edad osciló entre 19 y 64 años, con un promedio de 41 años (Tabla 1). El tratamiento quirúrgico más frecuente fue la reducción cerrada y colocación de fijadores. En el grupo de tratamiento no quirúrgico se incluyeron 76 pacientes manejados con aparato de yeso braquipalmar, el $38.7 \%$ con reducción y colocación de yeso y el $14.8 \%$ sin reducción previa a la colocación del yeso (Tabla 1).

Ambos grupos se evaluaron con la EFMM a 3 y 6 meses posteriores al tratamiento. Los resultados funcionales a los 3 meses se muestran en la figura 2. Se aprecia mejor funcionalidad notablemente a favor del tratamiento quirúrgico, tanto en el puntaje total como en cada uno de los dominios de la EFMM, si bien la significancia estadística solo se alcanzó en los dominios «Dolor», «Prensión» y «Puntaje total».

A los 6 meses (Fig. 3) se repitieron los resultados favoreciendo el manejo quirúrgico, destacando el aumento notable en los apartados «No trabaja» y «Desempleado» en el dominio «Estado laboral» de la EFMM, a expensas de los pacientes con manejo no quirúrgico, y se aprecia que la mayoría de los tratados quirúrgicamente regresaron a trabajar. En este corte, la mayoría de los pacientes no operados reportaron una funcionalidad (resultado total de la EFMM) regular o mala; en cambio, los que se sometieron a cirugía reportaron una funcionalidad regular, buena o excelente. Los mejores resultados con la cirugía son notables también en el dominio «Dolor». Sin embargo, 
Cirugía y Cirujanos. 2021;89(3)

Tabla 1. Resultados de los grupos, número de pacientes, sexo, edad, tipo de tratamiento y días de incapacidad

\begin{tabular}{|c|c|c|c|c|c|c|c|}
\hline \multicolumn{8}{|l|}{ Resultados } \\
\hline \multirow[b]{2}{*}{ Número de pacientes } & \multirow{4}{*}{$\begin{array}{l}\text { Mujeres } \\
\text { Hombres } \\
\text { Total }\end{array}$} & \multicolumn{2}{|c|}{ Grupo quirúrgico } & \multicolumn{2}{|c|}{ Grupo no quirúrgico } & \multicolumn{2}{|c|}{ Total } \\
\hline & & 25 & $12.8 \%$ & 29 & $20.4 \%$ & 54 & $34 \%$ \\
\hline & & 58 & $29.7 \%$ & 47 & $33.1 \%$ & 105 & $66 \%$ \\
\hline & & 83 & $52.2 \%$ & 76 & $47.8 \%$ & 159 & $100 \%$ \\
\hline \multirow[t]{5}{*}{ Edad (años) } & Mínimo & \multicolumn{2}{|c|}{19} & \multicolumn{2}{|c|}{21} & \multicolumn{2}{|c|}{19} \\
\hline & Máximo & \multicolumn{2}{|c|}{64} & \multicolumn{2}{|c|}{62} & \multicolumn{2}{|c|}{64} \\
\hline & Promedio & \multicolumn{2}{|c|}{40.18} & \multicolumn{2}{|c|}{42.5} & \multicolumn{2}{|c|}{41.2} \\
\hline & $\mathrm{DE}$ & \multicolumn{2}{|c|}{11.637} & \multicolumn{2}{|c|}{12.016} & \multicolumn{2}{|c|}{17.541} \\
\hline & \multicolumn{3}{|c|}{ Grupo quirúrgico } & \multicolumn{4}{|c|}{ Grupo no quirúrgico } \\
\hline \multirow[t]{6}{*}{ Tratamiento } & Fijador 57 & & $29.2 \%$ & \multirow{6}{*}{\multicolumn{2}{|c|}{$\begin{array}{l}\text { Reducción+yeso } 55 \\
\text { Yeso-reducción } 21 \\
\text { Modificadores } \\
\text { Dolor } 14 \\
\text { Edema } 5 \\
\text { No se modificó } 54\end{array}$}} & & \multirow{6}{*}{$\begin{array}{c}38.7 \% \\
14.8 \% \\
9.9 \% \\
3.5 \% \\
38 \%\end{array}$} \\
\hline & Fijador+clavillo 9 & & $4.6 \%$ & & & & \\
\hline & Placa bloqueada 17 & & $8.7 \%$ & & & & \\
\hline & & & & & & & \\
\hline & & & & & & & \\
\hline & & & & & & & \\
\hline \multirow[t]{5}{*}{ Días de incapacidad } & Mínimo & & 43 & & & 56 & \\
\hline & Máximo Promedio & & 244 & & & 198 & \\
\hline & DE & & 93 & & & 119 & \\
\hline & & & 46.253 & & & 33.661 & \\
\hline & \multicolumn{7}{|c|}{ t de Student: $4.152 P=0.001$} \\
\hline
\end{tabular}

DE: desviación estándar.

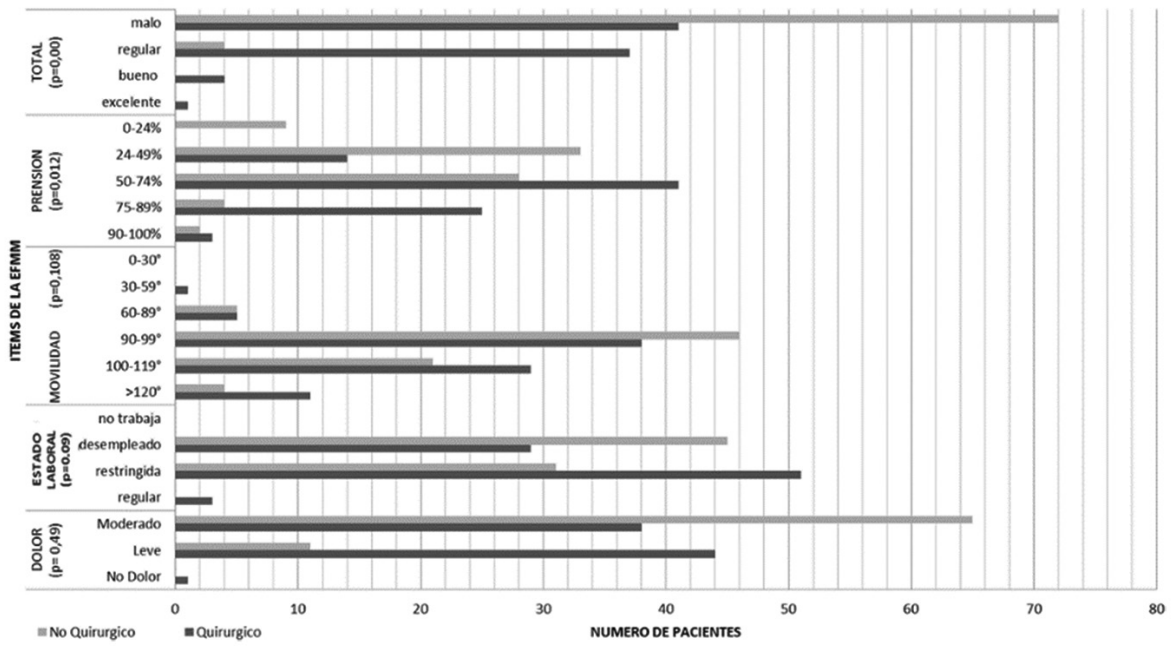

Figura 2. Comparación de la Escala Funcional de Mayo para Muñeca (EFMM) a 3 meses entre los grupos quirúrgico y no quirúrgico.

el valor de p solo resultó significativo en el dominio «Movilidad», el cual clínicamente es muy relevante.

También el promedio de días de incapacidad presenta una diferencia de 16 días menos para el grupo quirúrgico en comparación con el grupo no quirúrgico (Tabla 1).

\section{Discusión}

La fractura de radio distal es una de las más ampliamente estudiadas, con múltiples clasificaciones, cada una de acuerdo con parámetros radiográficos, anatómicos o del mecanismo de lesión. La 


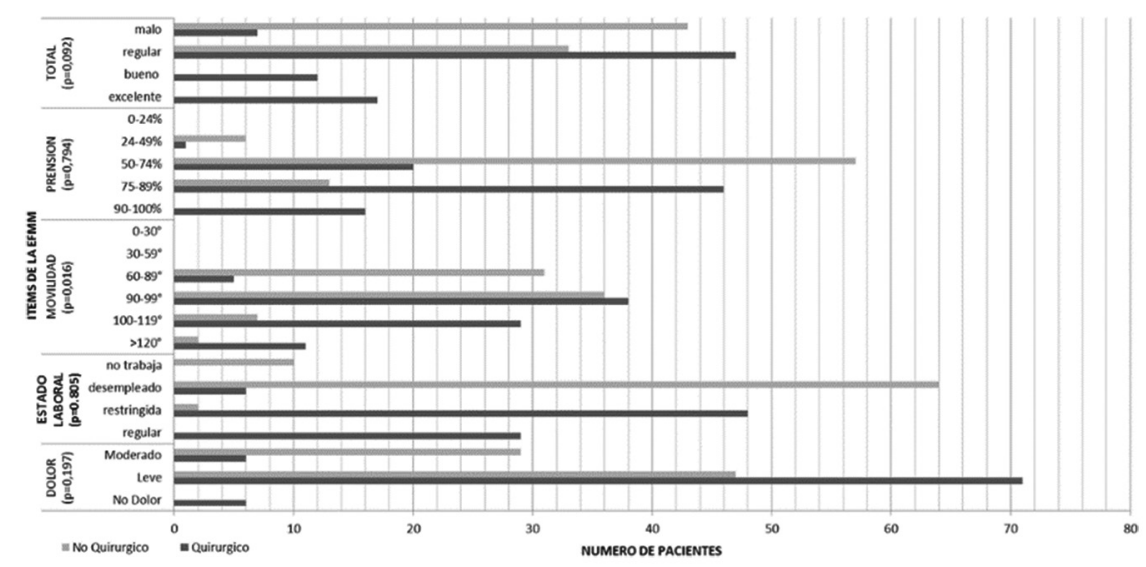

Figura 3. Comparación de la Escala Funcional de Mayo para Muñeca (EFMM) a 6 meses entre los grupos quirúrgico y no quirúrgico.

clasificación de la $\mathrm{AO}^{11}$, aunque de reciente auge, es puramente descriptiva respecto a la localización del trazo y no considera la biomecánica ni la cinemática de la lesión, que hacen más comprensibles los daños óseos y cápsulo-ligamentarios asociados a la fractura. Por esto, la clasificación más relevante, difundida y utilizada es la clasificación de Fernández (Fig. 1), que evalúa el mecanismo de lesión y los hallazgos anatómicos de la fractura. Las fracturas de tipo I o II generalmente reciben tratamiento no quirúrgico, y las fracturas de tipo IV y V son definitivamente de tratamiento quirúrgico ${ }^{3}$. Las fracturas de tipo III presentan una gran variabilidad en la conducta terapéutica a seguir, lo que genera resultados funcionales diversos en la muñeca afectada.

La incidencia de complicaciones en la población presentada fue acorde a lo reportado en la literatura internacional: hasta un $30-50 \%$ para el manejo conservador y menos del $10 \%$ para el tratamiento quirúrgico $4,6,14$.

Para evaluar la funcionalidad de la muñeca no existe una escala perfecta. La EFMM se basa en cuatro dominios ponderados cada uno en un $25 \%$ : dolor (en una escala de Likert: sin dolor, leve/ocasional, moderado, grave) y prensión (esta última comparando la prensión de la muñeca afectada con la contralateral), ambos con cierto grado de subjetividad; y estado laboral y rangos de movilidad (medida con un goniómetro). Se prefirió esta escala por ser específica para la muñeca, de menor subjetividad que otras (como la DASH [Disability arm-shoulder-hand] y la PRWE [Patient-Rated Wrist Evaluation]) y exigiendo su calificación más alta esencialmente el movimiento y la función normales. Además, permite un análisis más detallado y significativo en cuanto a sus dominios y a su puntación total20-22.

En este estudio, la valoración a los 3 meses de evolución mostró diferencia en los puntajes de la EFMM a favor del tratamiento quirúrgico, tanto en el total de la puntuación como al separar los dominios; sin embargo, solo se alcanzó significancia estadística en los dominios de «Dolor» y «Prensión» y en la puntuación total. Existen reportes previos con resultados similares ${ }^{23}$. Es importante mencionar que el mejor puntaje en los dominios individuales y el total de la EFM sí guardan relevancia clínica, a contrapelo de la significancia estadística.

El grupo quirúrgico presenta menor dolor y mejor prensión respecto al grupo no quirúrgico, pero la aportación principal del presente trabajo es que el movimiento temprano de la muñeca generó un impacto positivo en la reincorporación temprana de los pacientes a las actividades diarias, resultando en menos días de incapacidad en el grupo quirúrgico (Tabla 1). Ello es congruente con los resultados de la puntuación total de la EFMM a los 3 meses, y a los 6 meses en los dominios "Capacidad" y «Dolor", y en el puntaje total. Estos resultados reafirman lo encontrado en otros estudios ${ }^{16}$.

En cuanto a la funcionalidad, de acuerdo con la literatura internacional, las diferencias entre manejos a favor de la cirugía se mantienen hasta los 6 meses, pero parecen disminuir o incluso desaparecer después de ese tiempo ${ }^{24}$. Existen evidencias de que, después de 6 meses, la evolución funcional muestra una meseta independientemente del tratamiento 
realizado ${ }^{20}$. El presente estudio concuerda, pues la escala funcional a 6 meses no muestra diferencia significativa.

Otros estudios que comparan procedimientos quirúrgicos particulares muestran resultados variables $^{16,25-27}$, como al contrastar la instalación de placa volar en adultos mayores en comparación con inmovilización con yeso ${ }^{25}$, sin encontrar diferencias representativas en los rangos de movimiento y prensión. La población del presente trabajo, aunque es joven, registró similitudes respecto a movilidad a los 6 meses del tratamiento, pero mejor prensión en el manejo quirúrgico. Otro reporte compara la instalación percutánea de clavillos de Kirschner y manejo no quirúrgi$\mathrm{CO}^{16}$ sin encontrar diferencias entre ambos tratamientos, probablemente debido a la ausencia de un mejor implante, como placa o algún fijador.

La movilización temprana parece mejorar la funcionalidad, tanto en el presente estudio como en otros reportes $^{26}$ que no registran diferencias en el dolor, pero sí en cuanto a que la inmovilización prologada de más de 6 semanas en el grupo manejado de manera no quirúrgica provocó un impacto negativo en la funcionalidad, lo que concuerda con nuestro estudio.

Curtin y Hernández-Boussard ${ }^{27}$ reportan un grupo de pacientes en quienes el dolor fue la principal causa de reconsulta, asociado al tratamiento realizado $(8 \%$ posoperados con placa, $11 \%$ con fijadores externos y $14 \%$ con reducción cerrada); en el presente trabajo, el dolor presentó diferencia significativa entre los grupos a los 3 y 6 meses. Destaca en ambos cortes que la mayoría de los pacientes quirúrgicos reportan dolor leve o sin dolor, mientras que todos los de tratamiento conservador reportan dolor, al menos leve.

En la literatura internacional, los estudios son mayormente en pacientes de 60 años o más ${ }^{1,7,10}$; nuestro trabajo comprende no solo este grupo etario, sino también población trabajadora joven.

Por otro lado, la existencia de múltiples escalas funcionales obstaculiza el consenso en torno a una prueba que ayude a homogenizar los resultados en cuanto a la funcionalidad específica de la muñeca ${ }^{20,22}$.

El presente trabajo aporta información epidemiológica acerca de este tipo de fracturas en población trabajadora, además de objetivar los resultados de reincorporación al trabajo a favor del manejo quirúrgico y las ventajas de la movilización temprana. Con ello pretende ayudar a esclarecer el panorama respecto a las opciones terapéuticas y su impacto en la función de los pacientes con fractura del radio distal de tipo III de la clasificación de Fernández.

\section{Conclusiones}

El presente estudio sugiere que en las fracturas de radio distal de grado III de Fernández, el tratamiento quirúrgico ofrece mejores resultados funcionales a los 3 meses, sobre todo en cuanto al dolor y la prensión, y a los 6 meses en lo referente a la movilidad, en comparación con el tratamiento no quirúrgico. De la misma forma, la población a quien se realiza tratamiento quirúrgico se reincorpora a sus actividades laborales en menos días que la población manejada con aparato de yeso.

\section{Responsabilidades éticas}

Protección de personas y animales. Los autores declaran que para esta investigación no se han realizado experimentos en seres humanos ni en animales.

Confidencialidad de los datos. Los autores declaran que han seguido los protocolos de su centro de trabajo sobre la publicación de datos de pacientes.

Derecho a la privacidad y consentimiento informado. Los autores han obtenido el consentimiento informado de los pacientes y/o sujetos referidos en el artículo. Este documento obra en poder del autor de correspondencia.

\section{Conflicto de intereses}

Los autores declaran que no existe conflicto de intereses.

\section{Bibliografía}

1. Alluri R, Hill J, Ghiassi A. Distal radius fractures: approaches, indications, and techniques. J Hand Surg Am. 2016;41:845-54.

2. Lax-Pérez R, Vicent-Vera J, Picazo-Gabaldón B, Sánchez-Baeza MC, Díaz-Almodóvar JL. Factores pronósticos en el tratamiento de las fracturas de radio distal: comparación entre placa volar y fijador externo. Rev Esp Cir Ortop Traumatol. 2008;52:300-5.

3. Fernández D, Jupiter J. Fractures of the distal radius. 2nd ed. New York: Springer; 1996. p. 25-49.

4. Mathews A, Chung K. Management of complications of distal radius fractures. Hand Clin. 2015;31:205-15.

5. Padegimas EM, Ilyas AM. Distal radius fractures: emergency department evaluation and management. Orthop Clin N Am. 2015;46:259-70.

6. Macintyre N, Dewan N. Epidemiology of distal radius fractures and factors predicting risk and prognosis. J Hand Ther. 2016;29:136-45.

7. Obert L, Rey P, Uhring J, Gasse N, Rochet S, Lepage D, et al. Fixation of distal radius fractures in adults: a review. Orthop Traumatol Surg Res. 2013;99:216-34.

8. Celester-Barreiro G, Masip-Bilbao B, Prego-Bestilleiro A, Dozo-García J. Fracturas de la unidad radiocubital distal. Rev Iberoam Cir Mano. 2010;38:136-56.

9. Wolfe S, Pederson W, Kozin SH, Cohen M. Green's operative hand surgery. 7th ed. Philadelphia: Elsevier; 2016.

10. Cai L, Zhu S, Du S, Lin W, Wang T, Lu D, et al. The relationship between radiographic parameters and clinical outcome of distal radius fractures in elderly patients. Orthop Traumatol Surg Res. 2015;101:827-31.

11. Fricker R, Jupiter J, Kastelec M. Distal forearm. En: Krikler S, Colton C, editores. AO surgery reference. AO Foundation. (Consultado el 16/04/2020.) Disponible en: https://surgeryreference.aofoundation.org/ orthopedic-trauma/adult-trauma/distal-forearm.

12. Cherubino $P$, Bini A, Marcolli B. Management of distal radius fractures: treatment protocol and functional results. Injury. 2010;41:1120-6. 
13. Capo JT, Tan V. Atlas of minimally invasive hand and wrist surgery. Boca Raton: CRC Press; 2007.

14. Tang JB. Distal radius fracture: diagnosis, treatment, and controversies. Clin Plastic Surg. 2014;41:481-99.

15. Jariwala AC, Phillips AR, Storey PA, Nuttall D, Watts AC. Internal fixation versus other surgical methods for treating distal radius fractures in adults. Cochrane Database Syst Rev. 2014;(7):CD011212.

16. Handoll HG, Rajan M. Closed reduction methods for treating distal radial fractures in adults. Cochrane Database Syst Rev. 2003;(1):CD003763.

17. Serrano-de la Cruz MJ. Fracturas distales de radio. Clasificación. Tratamiento conservador. Rev Esp Cir Osteoarticular. 2008:236:141-54

18. Amadio PC, Berquist TH, Smith DK, Ilstrup DM, Cooney WP 3rd, Linscheid RL. Scaphoid malunion. J Hand Surg Am. 1989;14:679-87.

19. Fernández-León RA, Moreno-Palacios JA, Rodríguez-Oyaga A, Peces-González N, Ruiz-Molina D, García-Delgado I. Resultados funcionales en fracturas de extremidad distal de radio. Tratamiento quirúrgico con placa DVR o tratamiento ortopédico. Trauma Fund MAPFRE. 2011:22:86-90

20. Barker SL, Rehman H, McCullough AL, Fielding S, Johnstone AJ. Assessment following distal radius fractures: a comparison of 4 scoring systems, visual numerical scales, and objective measurements. J Hand Surg. 2016;41:219e224.
21. Souer JS, Lozano-Calderón SA, Ring D. Predictors of wrist function and health status after operative treatment of fractures of the distal Radius. J Hand Surg. 2008;33A:157.e1-8.

22. Slutsky DJ. Outcomes assessment in wrist surgery. J Wrist Surg. 2013;2:1e-3e.

23. Tan V, Bratchenko W, Nourbakhsh A, Capo J. Comparative analysis of intramedullary nail fixation versus casting for treatment of distal radius fractures. J Hand Surg. 2012;37:460-8.e1.

24. Chen Y, Chen X, Li Z, Yan H, Zhou F, Gao W. Safety and efficacy of operative versus nonsurgical management of distal radius fractures in elderly patients: a systematic review and meta-analysis. J Hand Surg. 2016;41:404-13.

25. Arora R, Lutz M, Hennerbichler A, Krappinger D, Espen D, Gabl M. Complications following internal fixation of unstable distal radius fracture with a palmar locking-plate. J Orthop Trauma. 2007;21: 316-22.

26. Aktekin CN, Altay M, Gursoy Z, Aktekin LA, Ozturk AM, Tabak AY. Comparison between external fixation and cast treatment in the management of distal radius fractures in patients aged 65 years and older. $J$ Hand Surg. 2010;35:736-42.

27. Curtin CM, Hernández-Boussard T. Readmissions after treatment of distal radius fractures. J Hand Surg. 2014;39:1926-32. 